\title{
Intermédialités
}

Histoire et théorie des arts, des lettres et des techniques

Intermediality

History and Theory of the Arts, Literature and Technologies

\section{Aimer une statue : Pygmalion ou la fable de l'amour comblé}

\section{Aurélia Gaillard}

Numéro 4, automne 2004

Aimer

Loving

URI : https://id.erudit.org/iderudit/1005477ar

DOI : https://doi.org/10.7202/1005477ar

Aller au sommaire du numéro

Éditeur(s)

Centre de recherche sur l'intermédialité

ISSN

1705-8546 (imprimé)

1920-3136 (numérique)

Découvrir la revue

Citer cet article

Gaillard, A. (2004). Aimer une statue : Pygmalion ou la fable de l'amour comblé Intermédialités / Intermediality, (4), 67-85. https://doi.org/10.7202/1005477ar
Résumé de l'article

L'amour pour les statues est apparemment un de ces sujets bizarres, une sorte d'hapax et une perversion : à partir de la fable de Pygmalion et de quelques autres histoires d'amours marmoréennes, je veux soutenir le paradoxe qu'aimer une statue est au contraire le scénario et la structure profonde de toute histoire d'amour comblé. Cette histoire-là d'une part démontre (ou révèle) le mécanisme de la rencontre amoureuse, d'autre part propose un grossissement des rôles et des postures de tout amoureux. La bizarrerie et la perversion seraient ainsi ce qui fonde l'acte même d'aimer : aimer, c'est toujours aimer une statue. 


\title{
Aimer une statue: Pygmalion ou la fable de l'amour comblé
}

\author{
Aurélia Gaillard
}

E n clin d'œil à Barthes, je commencerai par quelques fragments du discours amoureux d'une statue adressé à son créateur, Pygmalion : c'est donc une statue qui parle et qui dit: "Tout ce que je connais de moi / C'est que je vous adore $^{1}$.» Et c'est donc Pygmalion qui répond: «Ce jour a comblé tous mes vœux / Vous vivez, vous aimez et j’aime².»Voici apparemment le comble de l'amour comblé : faire que la conscience d'aimer soit conscience d'exister, faire coïncider la réciprocité du sentiment amoureux avec le flux de la vie, fonder l'existence sur l'amour.

Je vis, j'aime: cela n'appelle pas d'objet, c'est un double cri d'existence.

Vous vivez, vous aimez: cela n’est pas différent de la proposition précédente, car «vous», c'est «moi »; « moi », c'est «vous» : dans la fable de l'amour comblé, sujet et objet d'amour se confondent. Seul prédomine «le bonheur d'aimer et d'être ${ }^{3} »$.

«Moi», «Ce n'est plus moi», «Ah! encore moi»: le discours amoureux peut paraître plat: il est minimaliste et exclamatif, «Ah! » pourrait suffire. C'est le cri de la nature - celui que Rousseau appelle de ses vœux, c'est le premier

1. Antoine Houdar de La Motte, Le triomphe des arts [1700], dans Pygmalions des Lumières, anthologie présentée par Henri Coulet, Paris, Éditions Desjonquères, coll. "XVIII siècle", 1998, p. 44.

2. Jean-François de Saint-Lambert, Pygmalion [1769], dans Pygmalions des Lumières, p. 75 .

3. Jean-François de Saint-Lambert, Pygmalion, p. 75. 
langage de l'homme, "le plus universel, le plus énergique ${ }^{4}$ ", langage comme retrouvé du cri primitif et du geste imitatif. C'est un langage d'avant la belle langue, entre cri et soupir, le langage du corps - c'est la Galathée de Rousseau qui parle ici. «Moi», lorsqu'elle se touche; "plus moi », lorsqu'elle touche un (autre) marbre; "encore moi », lorsqu'elle touche le statuaire 5 . Ce n'est plus seulement le sujet et l'objet du désir qui se confondent alors, selon l'image biblique d'un sujet agrandi ( "c'est l'os de mes os / et la chair de ma chair ${ }^{6}$ »), c'est la distinction même qui fonde la connaissance de soi et du monde, distinction entre un sujet animé et un objet inanimé: sauf que cette distinction, dans l'amour, amène à fondre ensemble sujet animé et sujet aimé : les objets, ce sont les autres, tous les autres, le sujet aimant et aimé, c'est le couple amoureux. Car l'amour est d'abord mouvement (é-motion), conscience qui fait sortir le sujet de sa torpeur et donc transforme l'objet qu'il était (un bois, un marbre) en un sujet sensible - et même ultrasensible, émotif, exalté, enflammé: «je vis certainement, puisque j'en suis enivrée ${ }^{7} »$. Galathée n’a effectivement plus rien à voir avec ces marbres épars qui traînent encore dans l'atelier de l'artiste. En un instant, l'amour l'a transformée en palpitante maîtresse.

Je pourrais continuer longtemps à déployer la syntaxe et le scénario du discours amoureux de ce couple «bizarre» d'une statue et d'un statuaire: ce que je veux soutenir, à partir de la fable, exemplaire, de Pygmalion, telle qu'elle a été notamment repensée au XVIII ${ }^{\mathrm{e}}$ siècle $^{8}$, moment où le mythe antique se

4. Jean-Jacques Rousseau, Discours sur l'origine et les fondements de l'inégalité parmi les hommes, première partie, Jacques Roger (éd.), Paris, Éditions Garnier-Flammarion, 1971 [1754], p. 190.

5. Jean-Jacques Rousseau, Pygmalion, dans (Euvres complètes, Bernard Gagnebin et Marcel Raymond (éds.), Paris, Éditions Gallimard, coll. «Bibliothèque de la Pléiade», $195^{8}$ [composé pour la scène lyrique en 1762], t. II, p. 1230-1231.

6. Genèse, 2, 23.

7. André-François Boureau-Deslandes, Pygmalion ou la statue animée [1742], dans Pygmalions des Lumières, p. 64.

8. Je renvoie notamment aux études décisives de Hans Sckommadau, Pygmalion bei Franzosen und Deutschen in 18. Jahrhundert, Wiesbaden, Franz Steiner Verlag, 1970; Annegret Dinter, Der Pygmalion-Stoff in der europaïschen Literatur - Rezeptiongeschichte einer Ovid-Fabel, Heidelberg, Carl Winter Universistätsverlag, 1979 et de John L. Carr, "Pygmalion and the Philosophers. The Animated Statue in Eighteenth-Century France ", Journal of the Warburg and Courtauld Institutes, 1960, vol. XXIII, n ${ }^{\circ 5}$ 3-4, p. 239-255, ainsi qu'à mon ouvrage, Le corps des statues - le vivant et son simulacre à l'âge classique (de 
trouve actualisé par la question philosophique de l'animation de la matière et par toutes les reformulations issues du concept de corps-machine cartésien, c'est un paradoxe apparent: comment cet étrange scénario amoureux (aimer une statue) peut constituer non pas un hapax mais la trame, la fable, le comble de toute histoire d'amour comblé. En effet, le discours amoureux de ce couplelà (amoureux/statue) me semble rigoureusement semblable à tout autre: il ne fait que souligner et revivifier les images éculées des propos fades des histoires d'amour. Lorsque la statue «s'amollit» d'aimer et «s'anime», l'image d'un minéral en fusion, qui se liquéfie, vient rehausser le cliché; lorsque Pygmalion «devient fou», le surgissement du surnaturel, de la statue en marche, vient créditer l'expression figée. Les «réalités» de la condition du sujet amoureux, comme l'impression de dépendance, la déification de «l'objet» aimé, son culte, le sentiment de « déréalité ${ }^{9}$ ", tout cela trouve son expression symbolique dans la fable de l'amour pour les statues, qui dépasse d'ailleurs largement le cadre de l'histoire de Pygmalion, même revisitée par le XVIII ${ }^{\mathrm{e}}$ siècle: s'enfermer dans un tombeau ou la pièce d'une maison particulière pour se livrer à des amours marmoréennes (comme en atteste la tradition grecque ${ }^{10}$, ou encore, pour notre corpus, cette histoire dénichée par René Démoris ${ }^{11}$ de deux mousquetaires qui finissent par enlacer la statue de Vénus dont ils sont tombés amoureux dans le parc de Versailles ${ }^{12}$ ) peut ainsi être considéré comme une narrativisation des concepts de sacralisation ou de «déréalité », même si la «sacralisation » dans le cas de l'usage que le XVIII siècle (et notamment les philosophes matérialistes, Diderot ou Deslandes) fait du mythe de Pygmalion pose problème - j’y revien-

Descartes à Diderot), Paris, Éditions Honoré Champion, coll. «Les Dix-huitièmes siècles ", 2003, surtout les chapitres 2 à 4 de la seconde partie consacrés aux Pygmalions des Lumières (p. 87-135).

9. «Déréalité: sentiment d'absence, retrait de réalité éprouvé par le sujet amoureux, face au monde.» (Roland Barthes, Fragments d'un discours amoureux, Paris, Éditions du Seuil, coll. «Tel quel», 1977, p. 103)

10. Voir Anne Jacquemin, «Les tentations de la chair et du marbre», Cahiers du centre de recherche littéraires et historiques - Centre interdisciplinaire de recherches sur les civilisations et littératures du monde anglophone-afro-indianocéanique (Cahiers C.R.L.H.C.I.R.A.O.I.), $\mathrm{n}^{\circ}$ 2, «Pratiques du corps, médecine, hygiène, alimentation, sexualité », Université de La Réunion-Didier-Érudition, 1985, p. 13-21.

11. René Démoris, «Peinture et belles antiques dans la première moitié du siècle les statues vivent aussi », Dix-huitième siècle, n $^{\circ} 27,1995$, p. 129-142.

12. L'histoire, anonyme, s'intitule «La neuvaine de Cythère », dans L'art de plumer la plume sans crier, Cologne, Robert Le Turc, 1710. 
drai, il ne saurait bien sûr s'agir d'une «sacralisation » au sens d'un sacré divin mais plutôt d'une ritualisation qui reprendrait, comme par «citation », la structure ancienne (mythique ou biblique) de la chose sacrée et viendrait ainsi se saisir de la place d'un divin en train de se déliter dans un contexte de sécularisation. On peut penser bien sûr au concept de «divinisation à blanc » tel qu'il a été avancé par Jean Starobinski dans un article fondateur sur la fable et la mythologie aux XVII ${ }^{\mathrm{e}}$ et XVIII ${ }^{\mathrm{e}}$ siècles. Le renouveau paradoxal du recours à la mythologie antique à la fin du XVII ${ }^{\mathrm{e}}$ siècle, moment d'une critique aiguë du fabuleux (autour de la seconde Querelle des Anciens et Modernes), est l'objet d'un compromis (avec le sacré chrétien) et d'un déplacement: la dichotomie du sacré et du profane se trouve peu à peu redistribuée et supplantée par l'opposition raison/imagination. Le recours à la fable (et notamment à celle de Pygmalion) est donc souvent un recours limite: ainsi «la fable de Pygmalion figure, dans un langage encore mythique, une exigence d'expression de soimême, dont la prochaine manifestation consistera à refuser toute médiation mythique, tout recours à une fable préexistante ${ }^{13} »$.

Mon hypothèse, paradoxale, j’en conviens, est donc que ce couple-là ne raconte pas une histoire d'amour parmi tant d'autres, encore moins une déviance amoureuse, mais qu'il énonce ce qu'est la nature même d'aimer. Aussi, ce que met à nu ce couple amoureux, c'est finalement l'histoire même de l'amour, de tout amour: aimer une statue ou ce qu'aimer veut dire.

\section{LA RENCONTRE: LE RAVISSEMENT DÉCOMPOSÉ}

Tout au début de l'histoire, avant la rencontre amoureuse, avant le ravissement, il n'y a rien : l'atelier est chaos, un entassement de blocs. Le monde est minéral, insensible, les femmes (les Propœetides dans l'histoire de Pygmalion ${ }^{14}$ ) sont

13. Jean Starobinski, «Fable et mythologie aux XVII ${ }^{\mathrm{e}}$ et XVIII ${ }^{\mathrm{e}}$ siècles dans la littérature et la réflexion théorique", dans Dictionnaire des mythologies et des religions des sociétés traditionnelles et du monde antique, Yves Bonnefoy (dir.), Paris, Éditions Flammarion, 1981, t. 1, p. 396 (repris dans Le remède dans le mal. Critique et légitimation de l'artifice à l'âge des Lumières, Paris, Éditions Gallimard, coll. «NRF essais», 1989). On peut également consulter sur le sujet désormais bien défriché: Julie Boch, Les dieux désenchantés: la fable dans la pensée française de Huet à Voltaire, 1680-1760, Paris, Éditions Honoré Champion, 2002, et ma thèse, Fables, mythes, contes: l'esthétique de la fable et du fabuleux, 1660-1724, Paris, Éditions Honoré Champion, 1996.

14. Voir Ovide, Les métamorphoses, trad. Georges Lafaye, Paris, Éditions Les Belles Lettres, 1989, t. II (VI-X), livre X, v. 241 et suivants. 
doublement empierrées, elles ont le cœur endurci et, pour leurs fautes, sont changées en rochers, le seul homme (Pygmalion) est sculpteur : il vit de et dans la pierre et a aussi le cour pétrifié par une anaphrodisie qui fait de lui un homme sans femme. Ce monde dirait-on est de marbre - ou encore, de bois: froid, impassible, fermé, dense, compact, tourné vers l’intérieur, impénétrable; mais il est aussi veiné ou cerné, tacheté, ligneux, car ce monde n’est pas mort, il n'est pas encore né, ou plutôt pas encore éveillé. Le marbre (ou le bois, ou l'ivoire, ou l'albâtre qui sont les matériaux les plus cités pour les statues animées ${ }^{15}$ ) est déjà chair, il a toujours été chair, il en a l'incarnat un peu rosé, pour les chairs claires, et la veinure.

À la fois, donc, il n'y a rien et tout est déjà là, en attente, en sommeil.

Le geste - doigt et parole divine confondus - qui va éveiller ce monde, c'est celui du sculpteur et c'est, d'abord, celui d'un rapt et d'un viol. Avec son ciseau, il s'empare d'un bloc, taille le marbre, entaille la chair. Mais ce premier rapt, à peine signalé, est enfoui dans l'oubli dès que la statue est achevée: presque aucune version ne l'atteste (ou se contente d'un «il sculpta ${ }^{16} »$ ), la statue est toujours déjà là, parfaite icône, quand commence l'histoire. Néanmoins, le «rapt» continue: le statuaire palpe, malaxe, embrasse, serre contre

15. Le bois qui évoque plutôt l'idole archaïque (le xoanon des Grecs) ou les premières statues animées, celles façonnées par Dédale, en bois recouvertes de métal (voir Françoise Frontisi-Ducroux, Dédale, mythologie de l'artisan en Grèce ancienne, Paris, Éditions François Maspero, 1975) est encore présent dans l'imaginaire de la statue animée à la fin du XVII ${ }^{\mathrm{e}}$ et au XVIII ${ }^{\mathrm{e}}$ siècles mais seulement dans cette relation stricte à l'idole (chez Jean de La Fontaine bien sûr, dans la fable «L'homme et l'idole de bois », Euvres complètes, Jean-Pierre Collinet (éd.), Paris, Éditions Gallimard, coll. «Bibliothèque de la Pléiade», 1991, t. I, livre IV, fable VIII, p. 151, ou chez Bernard Le Bovier de Fontenelle, dans une églogue, La statue de l'amour, VII églogue, dans (Euvres complètes, Alain Niderst (éd.), Paris, Éditions Fayard, coll. «Corpus», 1991, t. 2, p. 361). Sinon, la plupart des Vénus ou Galathée qui apparaissent dans les reprises de l'histoire de Pygmalion à la même période sont en marbre (chez Deslandes ou chez Rousseau) ou encore en albâtre (dans Le nouveau Pygmalion de Jean Auguste Jullien dit Desboulmiers, en 1766, dans Pygmalions des Lumières, p. 95). Le célèbre groupe représentant Pygmalion et sa statue, «réel », celui-là, sculpté par Falconet, exposé au Salon de 1763 et commenté par Diderot est bien sûr en marbre (fig. 1-3). On peut toujours l'admirer au Musée du Louvre.

16. Ovide : «il sculpta dans l'ivoire à la blancheur de neige un corps auquel il donna une beauté qu'aucune femme ne peut tenir de la nature.» (Ovide, Les métamorphoses, t. II, livre X, v. 247-249) 
lui la statue inanimée. C'est une forme adoucie de viol, le coup de maillet s'est fait caresse, mais la statue est toujours inerte, non consentante puisque non consciente. Le geste est devenu ambigu: entre celui du sculpteur et celui de l'amoureux (qui palpe ou qui caresse?). Car tout amoureux est aussi sculpteur : la main qui «manie » le corps ${ }^{17}$ est celle de l'artiste; aimer c'est manipuler un corps pour en faire comme une œuvre d'art. Le rapt, au départ de toute histoire d'amour, est appelé « ravissement » (enamoration), c'est que l'amoureux est fondamentalement hypocrite (ou perdu, faible d'esprit): il prend la pose de l'idolâtre pour mieux dissimuler son iconoclastie. Le sculpteur est idolâtre (et fétichiste ${ }^{18}$ ) parce qu'il aime l'objet et contribue à faire de cet objet aimé, l'objet de tous les objets (un chef-d'œuvre) : il couvre la statue de baisers mais aussi de vêtements, de présents, "passe à ses doigts des bagues de pierres précieuses, à son cou de longs colliers ${ }^{19} »$. Puis, il lui fait faire «un beau lit où il couchoit avecque elle ${ }^{20} »$. Mais l'autre face, corollaire, de l'amour de l'objet, c'est le désir de briser l'objet, le rêve de pulvérisation. A l'ouverture du Rêve de d'Alembert, Diderot - pour les besoins d'une démonstration contestable, plus ludique que convaincante $^{21}$ - s'exalte à l'idée de pulvériser le chef-d'œuvre de Falconet (le groupe de Pygmalion, justement): "Je prends la statue que vous voyez, je la mets dans un mortier, et à grands coups de pilon...22"

Soudain, le statuaire n'ose plus frapper, il est «arrêté»: «Il prend son maillet et son ciseau, puis s'avançant lentement, il monte, en hésitant, les gradins de la statue qu'il semble n'oser toucher. Enfin, le ciseau déjà levé, il s'arrête ${ }^{23}$. » Il est arrêté, mais on peut penser que, simultanément, il jouit de la tentation, il ne tiendrait qu'à lui...

17. «Il recommence aussi-tost à la baiser, il luy touche aussi-tost le sein, et sentit que l'yvoire s'amollissoit; que sa dureté cedoit à ses doigts comme feroit de la cire que le Soleil amollit, et que la main qui la manie, trouve capable de toutes formes. » (Pierre Du Ryer, Les métamorphoses d'Ovide, Paris, Antoine de Sommaville, 1660, p. 440)

18. J'y reviendrai.

19. Ovide, Les métamorphoses, t. II, livre X, v. 263-264.

20. Pierre Du Ryer, Les métamorphoses d'Ovide, p. 439.

21. Il s'agit de démontrer la sensibilité de la matière par la transformation d'une statue en «chair», transformation obtenue par l'ingestion de cultures ayant poussé dans l'humus où a été pulvérisée la statue.

22. Denis Diderot, Le rêve de d'Alembert [1769], dans CEuvres philosophiques, Paul Vernière (éd.), Paris, Éditions Bordas, coll. «Classiques Garnier», 1990, p. 263.

23. Jean-Jacques Rousseau, Pygmalion, p. 1231. 


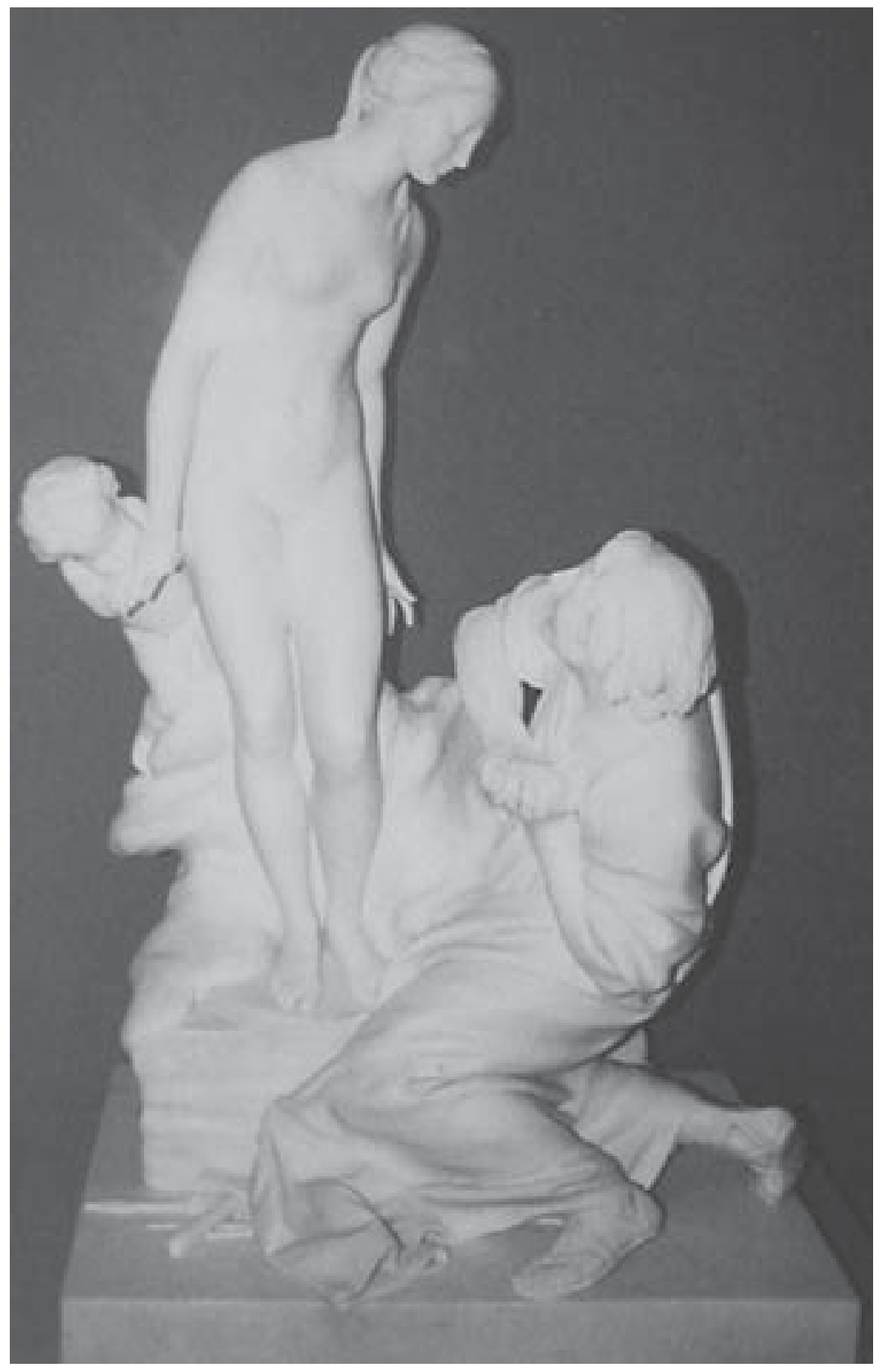

Fig. 1. Étienne-Maurice Falconet, Le groupe de Pygmalion, 1763, Paris, Musée du Louvre (cliché Aurélia Gaillard). 
Le «coup de foudre» amoureux, dans l'histoire de Pygmalion, n'est pas simultané: d'une part, le statuaire lui-même doit fabriquer son propre objet d'amour, d'autre part, la réciprocité du sentiment n'est pas une donnée mais une conquête. On peut dire que le moment de la rencontre est décomposé: comme si cette densité fulgurante du saisissement amoureux était ralentie à l'extrême et que se dévoilaient ainsi tous les états intermédiaires et très progressifs de «l'enamoration». Le statuaire est «vide», homme sans femme, homme du chaos, il crée un objet d'amour fait pour tomber amoureux, en tombe amoureux (le moment où le maillet est «arrêté »), est malheureux, éploré («Fatal amour! cruel vainqueur ${ }^{24}$ !») jusqu’à ce que le dispositif en miroir se mette en place: jusqu'à ce que la statue "vide» (pétrifiée, inconsciente, insensible) devienne sujet et sujet amoureux dans un même temps (être et aimer, c'est la même chose). «Elle vit celui qui l'aimait ${ }^{25}$ » : elle voit pour la première fois (par le regard et par la conscience, pour le XVIII ${ }^{\mathrm{e}}$ siècle, ce premier « regard » est un «toucher» qui seul peut constituer la première étape véritable de la connaissance ${ }^{26}$ ) et aime celui qu'elle voit. Il n'y a pas tant réciprocité que chiasme (elle voit ce qu'elle aime et aime ce qu'elle voit), c'est-à-dire que la jouissance d'aimer est inséparable de la jouissance de l'aliénation, de l'enfermement: le monde était un néant, ce néant désormais est surabondant, le vide s'est fait plein - à l'excès, mais plein d'un seul être, agrandi démesurément.

Ainsi, cette fameuse reconstitution d'une scène inaugurale de «coup de foudre » qui sert le plus souvent de fondement à toute histoire d'amour ${ }^{27}$ n'est pas ici donnée justement après-coup mais dans le présent de la rencontre: grossissement et ralentissement permettent alors de décomposer la scène.

24. Antoine Houdar de La Motte, Le triomphe des arts, p. 39.

25. Ovide, Les métamorphoses, t. II, livre X, v. 294.

26. Voir notamment Étienne Bonnot de Condillac dans le Traité des sensations qui réserve au toucher la place décisive dans l'acquisition des connaissances et la conscience de soi, et ceci à partir même de la fiction d'une statue, simulacre humain qui s'anime progressivement: Étienne Bonnot de Condillac, Traité des sensations, Paris, Éditions Fayard, coll. «Corpus», 1984 [1754].

27. «Il y a un leurre du temps amoureux [...], la scène initiale au cours de laquelle j'ai été ravi, je ne fais que la reconstituer : c'est un après-coup. Je reconstruis une image traumatique, que je vis au présent, mais que je conjugue (que je parle) au passé: “je le vis, je rougis, je pâlis à sa vue. Un trouble s'éleva dans mon âme éperdue": le coup de foudre se dit toujours au passé simple: car il est à la fois passé (reconstruit) et simple (ponctuel).» (Roland Barthes, Fragments du discours amoureux, p. 228) 


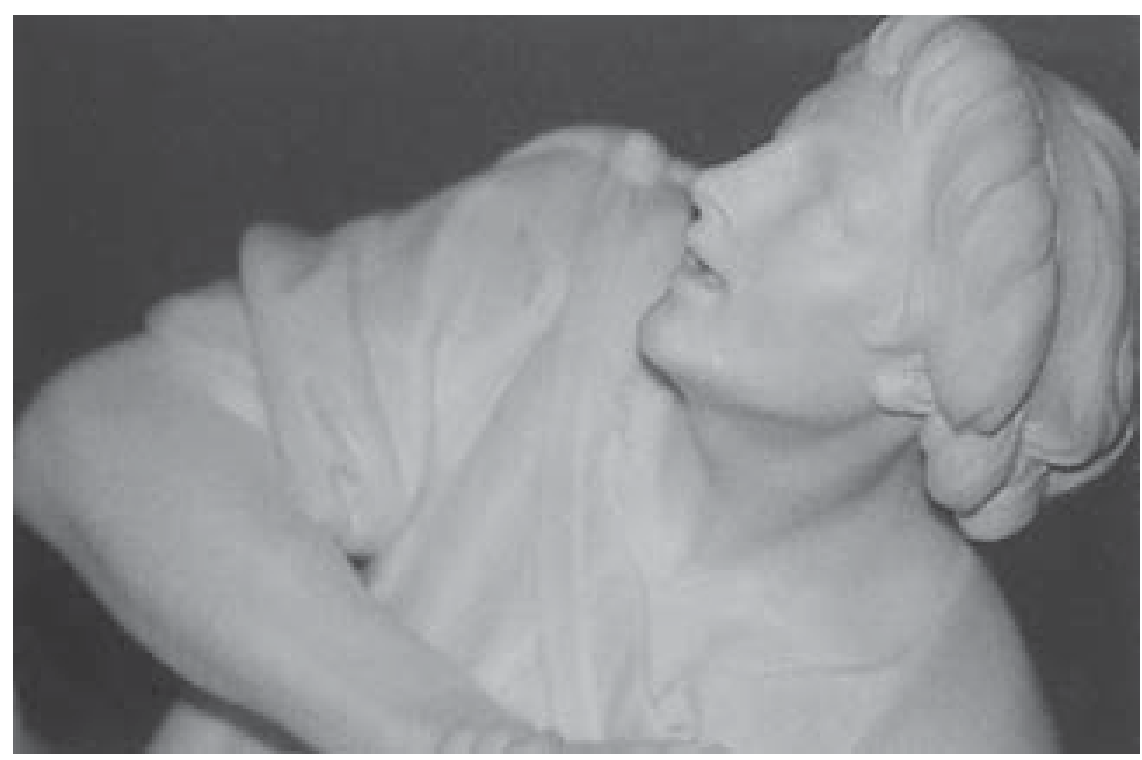

Fig. 2. Étienne-Maurice Falconet, Le groupe de Pygmalion (détail), 1763, Paris, Musée du Louvre (cliché Aurélia Gaillard).

Quand il reprend l'histoire de Pygmalion en 1742, le philosophe AndréFrançois Deslandes s'extasie devant un «sujet aussi bizarre et aussi philosophi$q^{2} e^{28}$ » «l'un n'est point contraire à l'autre », ajoute-t-il. Et la «leçon» philosophique qu'il tire précisément de cette histoire étrange c'est aussi celle d'une décomposition du mouvement trop rapide de la nature: l'animation de la matière, qui semble se faire "d'un coup», se fait, en vérité, par d'infimes modifications très progressives. On peut facilement tirer la même leçon pour le sentiment amoureux: ce «qu'aimer» semble faire d'un coup n'est que le résultat très progressif d'infinis changements. "Ce changement ne se fait point brusquement et par sauts: il se fait par degrés, par nuances, par des mouvements insensibles. Il y a un éloignement infini d'un état à l'autre; mais cet infini s'achève dans un temps très fini ${ }^{29}$.»

28. André-François Deslandes (dit Boureau-Deslandes), Pygmalion ou la statue animée, préface «À Madame la comtesse de G. », dans Pygmalions des Lumières, p. 49. 29. André-François Deslandes, Pygmalion, p. 59. 


\section{L'HISTOIRE: AIMER UNE STATUE}

Aimer une statue: évidemment, rien de plus bizarre en apparence.

Idolâtre: c'est sans doute le premier qualificatif auquel on pense, entaché de tradition biblique. Et je crois que même au XVIII ${ }^{\mathrm{e}}$ siècle et chez des auteurs aussi éloignés d'une pensée religieuse que peuvent l'être les philosophes matérialistes Deslandes ou Diderot, il persiste non pas une croyance idolâtre ni même peut-être une «arrière-pensée » religieuse, mais une structure mentale, imaginaire, de l'idole: la fable permettrait justement de mettre au jour ce qui n'est plus alors recevable depuis la grande critique du fabuleux de la Querelle des Anciens et des Modernes ${ }^{30}$, quelque chose qui, sans être du divin ni du sacré, viendrait néanmoins en prendre la place et en remplir la fonction: je ne crois pas que la fable au XVIII ${ }^{\mathrm{e}}$ siècle une fois «démythifiée » soit un pur ornement $^{31}$, je crois que l'idole et l'imaginaire de la statue animée qui en dérive conservent (et sans doute encore maintenant) à l'intérieur même d'une pensée critique et laïcisée ce qu'à défaut d'un terme plus approprié j’appellerai une forme de divin ou de sacré, une palpitation de l'ancienne croyance, une «structure » mythique.

Aimer une statue, c'est d'abord, donc, et encore au XVIII ${ }^{\mathrm{e}}$ siècle, même si le sens de l'idolâtrie n'est plus la même, être idolâtre.

Leurs idoles sont en or et argent

Fabriquées

à main d'homme

Elles ont une bouche

elles ne parlent pas

Elles ont des yeux qui ne voient pas

elles ont des oreilles qui n'entendent rien

Elles ont un nez mais ne sentent rien

30. Je rappelle la décisive affirmation de Fontenelle selon laquelle les mythes grecs n'étaient qu'un «amas de Chimères, de rêveries et d'absurdités [...], l'histoire des erreurs de l'esprit humain.» (Bernard Le Bovier de Fontenelle, De l'origine des fables, Paris, Brunet, 1724, p. 353, repris dans les (Euvres complètes, t. III, p. 187 et 202)

31. Ce que j’ai essayé de montrer dans ma thèse (Fables, mythes, contes) et dans la plupart de mes travaux. 


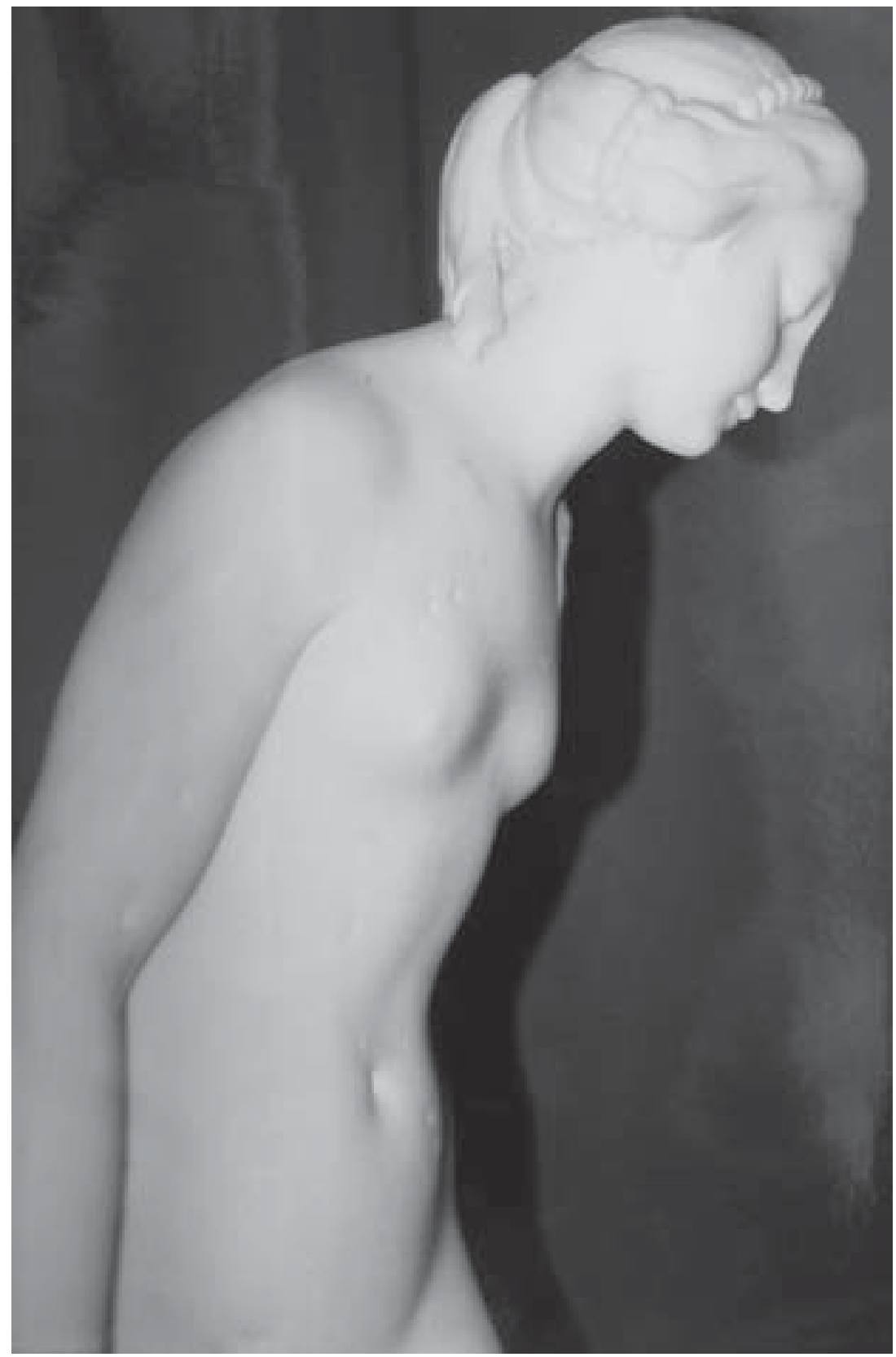

Fig. 3. Étienne-Maurice Falconet, Le groupe de Pygmalion (détail), 1763, Paris, Musée du Louvre (cliché Aurélia Gaillard). 
Avec leurs mains elles ne touchent rien

avec leurs pieds elles ne marchent pas

Pas un son

de leur bouche ${ }^{32}$.

En ce sens, c'est alors une aberration: mais au sens même où Jurgis Baltrušaitis use de ce mot, il s'agit non d'une erreur (aussi fascinante puisse-t-elle être), non d'une «pure» illusion, mais d'une vérité de fable ${ }^{33}$. Ainsi, dans la relation amoureuse, «l'objet» aimé est bien d'abord cette image parée d'or et d'argent, fabriquée de main d'homme, muette, immobile, sourde et aveugle. Il lui suffit d'être là, sa présence est réalité quand bien même elle ne serait ni sensible ni sensuelle. La statue, c'est la chose-même: une fin et une origine réunies dans un tout; chose et «cause » réunies, selon le rapprochement, par étymologie interposée (et en chiasme une fois encore) que propose Michel Serres: "Qu'est-ce qu'une statue? Une cause devenue chose et la cause des choses $^{34}$. » D'où la densité et le «bouclage» de la forme, ce qu'évoque, dans le psaume, la reprise à l'initial et au final d'une même variation sur le mutisme («Elles ont une bouche / elles ne parlent pas»; «Pas un son / de leur bouche»). Et c'est toujours cette palpitation sacrée ou mythique (faire comme si l'artifice était présence) qu'on retrouve dans la très belle et dense formulation d'un matérialiste athée comme Diderot: "La chose, c'est la statue seule, isolée, prête à se mouvoir ${ }^{35}$.» La statue, ainsi, est la figure même de l'objet d'amour: à distance, autonome, indifférente. Il faut, on l'a vu, pour tomber amoureux, forcer l'autre (forger puis forcer, forcer et forger simultanément). Il faut donc que l'autre résiste: mais la meilleure résistance, c'est encore l'ignorance. Elle est absente parce que « concentrée » et du même coup, elle absente à lui-même

32. «Psaumes ", 115, La Bible (nouvelle traduction), trad. Frédéric Boyer, Jean-Pierre Prévost, Marc Sevin, Paris, Montréal, Éditions Bayard, Médiaspaul, 2001, p. 1381.

33. "Les vérités métaphysiques sont les vérités des masques". Elles sont aussi les vérités des fables. Les illusions et les fictions qui naissent autour des formes répondent à une réalité et elles engendrent à leur tour des formes où les images et les légendes sont projetées et se matérialisent dans la vie.» (Jurgis Baltrušaitis, Aberrations: essai sur la légende des formes, 1. Les perspectives dépravées, Paris, Éditions Flammarion, coll. «Champs », 1995 [1983], p. 9-10)

34. Michel Serres, L'Hermaphrodite, dans Honoré de Balzac, Sarrasine, Paris, Éditions Garnier-Flammarion, 1989, p. 120.

35. Denis Diderot, Salon de 1765, Paris, Éditions Hermann, 1984 [1765], p. 285. 
le sujet amoureux. Aussi, la statue est-elle fascinante et immensément désirable parce qu'elle maintient à la fois une présence éclatante et une absence essentielle.

C'est le dispositif du piédestal: dans l'histoire de Pygmalion, le statuaire ne se contente pas de fabriquer de ses mains la statue, mais il lui façonne aussi, souvent, un piédestal qui souligne sa beauté, sa noblesse, et surtout son autonomie («seule, isolée»).

Pygmalion exalté, applaudi même de ceux qui étaient le plus fâchés qu’il eût si bien réussi, travailla à un piédestal de basalte ou marbre noir, veiné de rouge, afin d'y placer sa Vénus. Il la fit aussitôt transporter dans un salon isolé, qui était au bout de son jardin $[\ldots]$. Ce salon était peint en vert et or, et des lits de repos, un peu éloignés les uns des autres, offraient des asiles sûrs et commodes qui aidaient à la rêverie. Une lumière douce s'y répandait par quatre fenêtres garnies de feuilles de talc, et l'on diminuait encore le jour par des rideaux faits de peaux d'Espagne, qui se tiraient avec des cordons or et vert ${ }^{36}$.

Le piédestal n'est alors que le premier élément d'une installation qui renforce l'isolement constitutif de la statue et rappelle la structure sacrée de l'idole dressée au fond du temple, dans un saint des saints: mais cette «sacralisation » (dans le sens tout relatif que je lui ai donné plus haut) est aussi une théâtralisation - qui partiellement d'ailleurs la désacralise. L'installation ensuite se fait effectivement mise en scène, comme en témoigne notamment le jeu tout théâtral des lumières ( « et l'on diminuait encore le jour... ») et le souci extrême du décor (les matériaux, les couleurs mais aussi la disposition étudiée des lits de repos «un peu éloignés les uns des autres»).

Une autre variation sur l'histoire de Pygmalion, celle proposée par Desboulmiers en 1766 dans son Nouveau Pygmalion ${ }^{37}$, pousse la mise en scène à son extrême en inventant un dispositif fait de poulies et de machines, plus souvent utilisé dans les scènes libertines ${ }^{38}$ : la statue est bien dressée, grandeur

36. André-François Deslandes, Pygmalion, p. 57.

37. Le nouveau Pygmalion [1766], histoire véritable de Jean Auguste Jullien dit Desboulmiers, dans Pygmalions des Lumières, p. 77-98), trouve la justification de son titre dans son dénouement qui met en scène une véritable statue : le héros, tendre, Vermansai s'éprend d'une jeune comtesse, $\mathrm{M}^{\mathrm{me}}$ d'Orgeville, et l'idolâtre au point de dissimuler et de vénérer, dans son cabinet, une statue la représentant.

38. Voir notamment les travaux d'Henri Lafon, d'abord Les décors et les choses dans le roman français du dix-huitième siècle de Prévost à Sade, Oxford, The Voltaire Foundation, 
nature, sur un piédestal, confiné dans un cabinet secret mais il s'agit alors d'un truquage qui, tout en conférant à la statue réunie à son socle par l'artifice un caractère d'idole sacrée, dévoile néanmoins la «machinerie».

Vermansai, après avoir ouvert cette porte, presse deux boutons, et au moyen de balanciers et de machines dont on entendait aisément l'effet, il sort un piédestal du plancher d'en-bas, et il descend en même temps de celui d'en-haut une statue d'albâtre, ornée de festons et de guirlandes de roses ${ }^{39}$.

La référence théâtrale est ici tout à fait explicite : la statue, qui «descend d'enhaut », est bien un deus ex machina et là encore la «sacralisation » est interrogée - soulignée, renforcée mais aussi mise à distance ironique - par la théâtralisation.

Idolâtre et (presque) lucide: de la même façon, le sujet amoureux est la dupe, sans l'être, de son idolâtrie: il occupe successivement, et même parfois simultanément, tous les rôles, passif et actif, celui du spectateur transi et celui de metteur en scène; aimer, c'est ainsi façonner sa propre idole et oublier (ou suspendre un temps sa mémoire) qu'on l'a soi-même façonnée.

Chez Rousseau, même disposition avec d'infimes variations: le piédestal est «petit» mais «exhaussé par un gradin de marbre, formé de quelques marches demi-circulaires ${ }^{40} »$; quant au caractère sacré, il est évoqué et aussi souligné, affiché (à la différence de chez Deslandes, bien sûr, mais on connaît la différence des positions religieuses de l'un et l'autre ${ }^{41}$ ) non par un isolement mais par un voile qui recouvre entièrement la statue: «Je ne sais quelle émotion j'éprouve en touchant ce voile; une frayeur me saisit; je crois toucher au sanctuaire de quelque Divinité... ${ }^{42}$ »

coll. «Studies on Voltaire and the Eighteenth Century», 1992, mais aussi son étude «Machines à plaisir dans le roman français du XvIII ${ }^{\mathrm{e}}$ siècle ", Revue des sciences humaines, «La machine dans l'imaginaire (1650-1800)", Lille-3, $\mathrm{n}^{\circ \mathrm{s}} 186-187$, 1982-3, p. 111-121.

39. Desboulmiers, Le nouveau Pygmalion, p. 95.

40. Jean-Jacques Rousseau, Pygmalion, p. 104.

41. Je renvoie pour Rousseau notamment à la célèbre réfutation du matérialisme («les premières causes du mouvement ne sont point dans la matière...») et à son corollaire, l'affirmation d'une puissance divine ( «Cet être qui veut et qui peut, cet être actif par lui-même, cet être, enfin, quel qu'il soit, qui meut l'univers et ordonne toutes choses, je l'appelle Dieu»), qu'on trouve au cœur du livre IV de l'Émile (Profession de foi du vicaire savoyard, Paris, Éditions Garnier Flammarion, 1996 [1762], p. 62 et p. 68).

42. Jean-Jacques Rousseau, Pygmalion, p. 104. 
Mais l'autre versant de l'idolâtrie, c'est aussi l'iconoclastie et le sacrilège: aimer, c'est aussi profaner. Idolâtrer (l'amoureux est un prosélyte, tout juste converti à la nouvelle religion de l'amour) pour mieux profaner. La statue est toujours dressée : elle porte la mémoire des primitives pierres levées, elle inverse les rôles amoureux aussi. C'est elle qui est érigée, le statuaire, lui, se «prosterne $^{43}$ ». On le sait, la fascination est castration (le fascinus à la fois tendu et arraché de soi), aimer une statue, ce serait bien alors le comble de l'amour, un sommet qui est aussi un trop-plein, un point de bascule qui programme son possible retournement, bref, le moment aberrant où le désir exacerbé est comme maintenu hors désir.

Mais cette «aberration » est-elle perversion? Aimer une statue, est-ce une déviance?

D'abord: concernant l'amour pour les statues ou les simulacres, le récit des grandes «déviances» (fétichisme, masochisme, nécrophilie) se fera plutôt à partir du XIX siècle : je pense bien entendu à Wanda, femme et statue, «Vénus à la fourrure» qui suscite fétichisme et masochisme ${ }^{44}$, à l'amour obsessionnel d'un Hanold pour un bas-relief de pierre représentant un pied dans l'élan de la marche dans le récit de Jensen, Gradiva, et au commentaire minutieux qu'en fait Freud ${ }^{45}$, ou à la passion de Romuald pour Clarimonde morte dans la Morte amoureuse de Gautier ${ }^{46}$ - mais les exemples notamment autour du conte fantastique encore chez Gautier comme chez Hoffmann, Villiers de l'Isle-Adam,

43. Jean-Jacques Rousseau, Pygmalion, p. 104.

44. «Souvent, aussi, la nuit, je rends visite à ma bien-aimée froide et cruelle, et je reste à ses genoux, le visage pressé contre la pierre glacée où sont posés ses pieds, et je l'adore. » (Léopold Von Sacher-Masoch, La Vénus à la fourrure, trad. Aude Willm, Paris, Éditions mille et une nuits, 1999 [1870], p. 24)

45. Sigmund Freud, Le délire et les rêves dans la Gradiva de W. Jensen, précédé de Gradiva de Wilhelm Jensen, trad. Paule Arhex et Rose-Marie Zeitlin, Paris, Éditions Gallimard, coll. «Folio/Essais», 1986 [1907].

46. «Elle avait pour tout vêtement le suaire de lin qui la recouvrait sur son lit de parade, dont elle retenait les plis sur sa poitrine, comme honteuse d'être si peu vêtue, mais sa petite main n'y suffisait plus; elle était si blanche, que la couleur de la draperie se confondait avec celle des chairs sous le pâle rayon de la lampe. Enveloppée de ce fin tissu qui trahissait tous les contours de son corps, elle ressemblait à une statue de marbre de baigneuse antique plutôt qu'à une femme douée de vie. » Ce qu'aime ainsi Romuald en Clarimonde morte, ou mort-vivante, ce n'est pas son improbable «résurrection », ce n'est pas la vivante, mais la statue (Théophile Gautier, La morte amoureuse, Paris, Éditions Gallimard, coll. «Folio», 1981 [1836], p. 102. Je souligne). 
Blackwood, etc. peuvent être aisément multipliés. Pour le XVIII siècle, la « déviance» reste souvent implicite, voilée ou «gazée» par le langage: mais l'on sait bien que le voile dénude plus qu'il n'habille, rehausse et souligne la nudité. L'Encyclopédie de Diderot et d'Alembert évoque ainsi ces perversions sous l'appellatif gazé de «faiblesses humaines»: le rédacteur de l'article «Statues des Grecs et des Romains », le chevalier de Jaucourt, cite une longue suite d'auteurs (Élien, Pline, Valère Maxime, Athénée, Plutarque, Clément d’Alexandrie) dont les récits sont «remplis d'exemples de ces faiblesses humaines pour les statues de Vénus qu'on voyait à Cnide et dans l'île de Chypre ${ }^{47}$ ». Le discours du XVIII ${ }^{\mathrm{e}}$ siècle est bien sûr moral et ici même «moralisateur » (il s'agit d'illustrer les passions de l'âme et ici les passions violentes, néfastes) : le seul exemple développé par Jaucourt (le rédacteur de l'article), celui, emprunté à Élien, d’un jeune Athénien qui n’arrive pas à acheter une statue de la Bonne-Fortune dont il est tombé amoureux et qui finit par se tuer, est alors au rebours de la tradition antique où l'aventure amoureuse avec les statues semblait plutôt heureuse ${ }^{48}$.

C'est surtout, après 1760, dans les développements plus libres, à partir du mythe de Pygmalion, dans ces «nouveaux Pygmalion", selon les titres de Desboulmiers (1766) ou de Rétif de la Bretonne ${ }^{49}$ (1780), que les implications et prolongements pervers de l'amour pour une femme-statue (ou statue-femme) sont développés.

Fétichisme, bien sûr: parce qu'il s'agit de l'amour de l'objet, ou même d'une partie de l'objet: l'amour pour la statue est alors une forme de fétichisme: le désir porte sur un fragment, une partie, un objet. Dans un récit de 1775 de Baculard d'Arnaud, Liebman - histoire allemande ${ }^{50}$, qui combine le

47. Denis Diderot et Jean Le Rond d'Alembert, Encyclopédie ou dictionnaire raisonné des sciences, des arts et des métiers, nouv. imp., fac-simile de la $1^{\mathrm{re}}$ édition, 1751-1780, Stuttgart-Bad Cannstatt, Friedrich Frommann Verlag, 1967, 35 tomes.

48. Voir Anne Jacquemin, "Les tentations de la chair et du marbre», p 13-21. Voir également l'analyse par Michel Foucault d'un texte du Pseudo-Lucien (Les amours, 16) qui raconte l'histoire d'un jeune homme épris, à Cnide, de la statue d'Aphrodite de Praxitèle avec laquelle il réussit à avoir une relation sexuelle mais en la prenant «en garçon» (Michel Foucault, Histoire de la sexualité, 3. Le souci de soi, Paris, Éditions Gallimard, coll. «Bibliothèque des histoires", 1984, p. 243-261).

49. Nicolas-Edme Rétif de la Bretonne, Le nouveau Pygmalion [1780], dans Pygmalions des Lumières, p. 173-207.

50. Baculard d'Arnaud, Liebman - histoire allemande [1775], dans Pygmalions des Lumières, p. 117-171. 
motif de «l'enfant de la nature ${ }^{51}$ » et l'histoire de Pygmalion, en mettant en scène un homme qui élève une petite fille (de sa naissance à l'âge adulte) pour en faire sa femme, différentes pulsions sont suggérées: fétichisme mais aussi nécrophilie et inceste. De fait, la fragmentation du corps de la jeune Amélie («l'enfant de la nature») frôle souvent le fétichisme: l'adoration porte sur des parties successives du corps (mains, pieds, genoux, visage) et le récit en donne souvent une vision parcellaire: «Je garde le silence sur mon projet, et me contente de me jeter à ses pieds, en couvrant sa main de baisers et de larmes délicieuses ${ }^{52}$.»

Nécrophilie aussi : aimer une statue, c'est aussi aimer, non pas le cadavre décomposé et la chair pourrissante mais la blanche et froide morte, sculpturale, diaphane. Toujours dans Liebman, le récit s'ouvre et se ferme sur une scène funèbre: «Un homme penché sur ce monument funèbre, de temps en temps l'embrassait avec plus de transport, et en poussant de ces gémissements sombres $[\ldots]^{53} »$. A défaut du corps de la morte, c'est la pierre tombale, métonymie de la femme-statue, qui est enlacée: la pulsion nécrophile tend aussi à abolir les limites entre le vivant et le mort, à déplacer et rendre perméable la frontière du vivant: «Lorsque j’embrasse ce tombeau qui contient sa cendre, je crois éprouver un tressaillement; la sensibilité serait-elle entièrement éteinte chez les morts $^{54}$ ?»

Cette fascination pour les cercueils et les mortes amoureuses, qui va, bien entendu, se déployer à la fin du XVIII ${ }^{\mathrm{e}}$ siècle et au tournant du siècle suivant, dans le genre sombre et la nouvelle tragique remise au goût du jour, en Angleterre d'abord, puis en France, rencontre alors avec le motif de la femme-statue façonnée par un maître (souvent père aussi ou tuteur, comme chez Baculard d'Arnaud mais surtout chez Rétif et chez Sade, dans Eugénie de Franval) un champ fantasmatique particulièrement propice à toutes sortes d'expérimentations: à la fin du récit sadien, le libertin Franval qui a éduqué sa fille selon ses

51. Voir Jean-Michel Racault, «Le motif de "l'enfant de la nature" dans la littérature du XVIII ${ }^{\mathrm{e}}$ siècle ou la recréation expérimentale de l'origine », dans Primitivisme et mythe des origines dans la France des Lumières 1680-1820, Paris, Presses universitaires de la Sorbonne, 1989, p. 101-117, ainsi que, du même auteur, Nulle part et ses environs voyage aux confins de l'utopie littéraire classique (1657-1802), Paris, Presses universitaires de la Sorbonne, 2003 .

52. Baculard d'Arnaud, Liebman, p. 136.

53. Baculard d'Arnaud, Liebman, p. 114.

54. Baculard d'Arnaud, Liebman, p. 168. 
propres principes, dénués des "préjugés » de la société et en a fait sa maîtresse, meurt sur les corps ensevelis de ses deux victimes (son épouse et sa fille) et à la fois féconde et flétrit (sang et sperme étant assimilés) les cadavres : "Son sang impur coule sur la victime et semble la flétrir bien plus que la venger ${ }^{55}$.»

Inceste: la pulsion incestueuse est directement liée à la confusion des rôles : l'amoureux veut être tout, le maître, mais aussi l'esclave ${ }^{56}$, le père, l'amant. M. De $\mathrm{M}^{\text {***; }}$, le héros du Nouveau Pygmalion de Rétif (1780), tombe amoureux de la fillette qu'il a fait élever pour la sauver de la pauvreté tandis qu'elle avait douze ans et tous les traits d'une nouvelle Cendrillon (elle «ramassait des cendres au pied d'une borne $\left.{ }^{57} »\right)$ : rapidement, la confusion des rôles s'installe clairement explicitée par le héros lui-même: «Tu es mon ouvrage; c'est moi qui t'ai créée, pour ainsi dire; je t'aime en père, en frère, en amant ${ }^{58}$. »Chez Rétif (dont on connaît l'importance du thème incestueux dans l'œuvre) de même que chez Sade, dans Eugénie de Franval en 1800, l'inceste (encore partiel du protecteur pour sa pupille chez Rétif et véritable, du libertin Franval pour sa fille Eugénie, chez Sade) est décrit non comme une «déviance» du comportement amoureux mais bien comme le comble même de l'amour comblé : c'est la société qui est déviante en ne reconnaissant pas cet amour «parfait» puisque tout en conservant une distinction des sexes, il s'agit bien d'un amour du même au même. Ce sont les seules bienséances qui inquiètent M. De $M^{* * * * *}$ chez Rétif: «Voilà mon embarras! En faire ma femme, est certainement le plus noble, peut-être le plus raisonnable; mon bonheur y est attaché. Mais ma famille! que dirait-on dans le monde? à la Cour ${ }^{59}$ ? » Chez

55. Donatien Alphonse François, marquis de Sade, Eugénie de Franval, dans Les crimes de l'amour, Michel Delon (éd.), Paris, Éditions Gallimard, coll. «Folio », 1987 [1800]. Comme pour Liebman de Baculard d'Arnaud, si le récit ne porte pas la mention de «nouveau Pygmalion », la référence au mythe est néanmoins explicite dans le texte: «Ah! mon ami, la folie de Pygmalion ne m’étonne plus...» (Marquis de Sade, Eugénie de Franval p. 315) Sans compter la scène centrale où Eugénie, nue, est «statufiée », c'està-dire présentée au double regard de son père et d'un autre libertin Valmont, sur un piédestal tournant. (Marquis de Sade, Eugénie de Franval, p. 348-349)

56. Bien qu'ayant dirigé en maître absolu l'éducation d'Amélie, Liebman s'écrie: «Liebman est ton amant, ton esclave; tu es ma maîtresse absolue. » (Baculard d'Arnaud, Liebman, p. 125)

57. Rétif de La Bretonne, Le nouveau Pygmalion, p. 175.

58. Baculard d'Arnaud, Liebman, p. 184.

59. Baculard d'Arnaud, Liebman, p. 188. 
Sade, Eugénie a dépassé son père et maître en libertinage: elle anticipe ses désirs les plus excessifs et comprend mal la nécessité de déguiser ses pulsions ressenties justement comme des «preuves d'amour» d'un corps libéré des contraintes d'une société frileuse. Dans les deux récits, l'inceste est l'idéal de l'amour.

Dès lors, les scénarios de toutes ces amours marmoréennes n'apparaissent plus que comme autant de manières d'aimer, sans doute amplifiées, intensifiées, voire radicalisées jusqu'au plus scandaleux et insoutenable mais néanmoins déjà présentes dans toute relation amoureuse.

Alors, aimer une statue, une bizarrerie perverse? Sans doute, mais le récit de cette perversion est sans nul doute le plus banal qui soit: pas d'amour sans perversion, c'est-à-dire sans détournement du désir normé, pas d'amour sans comportement asocial, pas d'amour sans surenchère, sans extravagance, sans bizarrerie. Pas d'amour sans statue à aimer. 